\title{
THE ROLE OF NORADRENERGIC AND SEROTONERGIC SYSTEMS IN THE HIPPOCAMPAL KINDLING EFFECT
}

\author{
Hiroaki ARAKI, Hironaka AIHARA, Shigenori WATANABE*, \\ Hisashi OHTA*, Tsuneyuki YAMAMOTO* and Showa UEKI* \\ Department of Pharmacology, Research Laboratories, Taisho Pharmaceutical Co., Ltd., \\ Saitama 330. Japan. "Department of Pharmacology. Faculty of Pharmaceutical Sciences. \\ Kyushu University, Fukuoka 812, Japan
}

Accepted August 21, 1982

\begin{abstract}
The role of the noradrenergic and the serotonergic systems in the development of hippocampal and amygdaloid kindling was studied. Dorsal noradrenaline bundle lesions markedly facilitated the formation of hippocampal and amygdaloid kindling. The most significant facilitation was observed in the earliest phase of kindling. Cortical noradrenaline content decreased by about $70 \%$ after dorsal noradrenaline bundle lesions. Midbrain raphe nuclei (both dorsal and medial raphe nuclei) lesions did not have any effect on the formation of both hippocampal and amygdaloid kindling. These results indicate that the dorsal noradrenaline bundle system plays an inhibitory role in the development of the seizure discharges and the behavioral convulsions in hippocampal and amygdaloid kindling.
\end{abstract}

Repeated low level electrical stimulation of the limbic structures in the brain produces behavioral convulsions and eventually leads to generalized full seizure (1). This phenomenon is called the kindling effect. It has been demonstrated that this model has several advantages over previously developed experi mental models of epilepsy $(2,3)$.

It has become clear that the brain catecholamines are of considerable importance in the normal suppression of seizure activity (4). In kindling, several experiments indicate that brain catecholamines play an inhibitory role in the development of seizure discharge and behavioral convulsions. Administration of reserpine (5), which depletes catecholamines or 6-hydroxydopamine (4-6) that destroys catecholamine nerve terminals, has been found to facilitate amygdaloid kindling in rats. The lesions of the stria terminalis accelerate amygdaloid kindling (7). Ehlers et al. (8) recently demonstrated that the depletion of forebrain noradrenaline (NA) by transection of the ascending NA pathway facilitates amygdaloid kindling in rats. Furthermore, previous research has suggested that the brain serotonin (5-HT) system also plays an important role in the epileptiform seizure activity (9). In the kindling, electrical stimulation (10) and lesion of the midbrain raphe nuclei (11, 12), blocked and facilitated amygdaloid kindling, respectively. Most of the studies on kindling effect has so far been done in the amygdala and cortex (13). However, hippocampal kindling has been studied less thoroughly. It is well known that afterdischarges are easily induced by electrical stimulation in the hippocampus (14) and the hippocampus as well as the amygdala is considered to be one of the foci in the psychomotor seizure (15)

The present experiment was undertaken to investigate the role of brain NA and $5-\mathrm{HT}$, by producing lesions in the dorsal NA bundle 
or midbrain raphe nuclei, in the formation of the hippocampal kindling effect.

\section{Materials and Methods}

Animals: Male Wistar strain rats, weighing 280-320 g at the time of surgery. were maintained and housed in an air-conditioned room at $22 \pm 1{ }^{\circ} \mathrm{C}$ with a $12 \mathrm{hr}$ light-dark schedule (lights on at 7:00). Food and water were given ad libitum during the experimental period.

Surgery and experimental procedure: The animal's head was fixed in a stereotaxic instrument under pentobarbital- $\mathrm{Na}(50 \mathrm{mg} /$ kg i.p.) anesthesia, and bipolar stainless steel electrodes (tip diameter $0.2 \mathrm{~mm}$, uninsulated length $0.5 \mathrm{~mm}$. polar distance $0.5 \mathrm{~mm}$ ) were chronically implanted in the hippocampus [anterior (A): $3.2 \mathrm{~mm}$, lateral (L): $2.8 \mathrm{~mm}$. ventral $(V): 2.0 \mathrm{~mm}$ ], amygdala [A: 5.4. L: $4.0, V:-2.5]$, reticular formation $[A: 1.5, L$ : 1.5. $V:-1.5]$ and the frontal cortex according to De Groot's brain atlas (16). For the bilateral dorsal NA bundle or the raphe nuclei lesions. an unipolar electrode made of a $0.2 \mathrm{~mm}$ diameter stainless steel wire which was insulated except for the tip was inserted into the brain according to the stereotaxic atlas of Konig and Klippel (17). The coordinates for the dorsal bundle were $\mathrm{A}: 1.3, \mathrm{~L}: 0.7, \mathrm{~V}$ : -1.0 ; those for the dorsal raphe nuclei were A: $0.3, L: 0.0, V:-0.8$ : and those for the medial raphe nuclei were $A: 0.3, L: 0.0, V$ : -2.8 . The dorsal NA bundle and the raphe nuclei lesions were made by applying an anodal current of $2 \mathrm{~mA}$ for $10 \mathrm{sec}$ and $3 \mathrm{~mA}$ for $15 \mathrm{sec}$, respectively. Sham operations were performed in the same manner without applying electrical current.

Following the chronic implantation of electrode and the dorsal NA bundle or raphe nuclei lesions, 2 weeks were allowed for recovery from the surgery before commencing the experiments. The animals were moved to an open-topped Plexiglas
$(38 \times 40 \times 45 \mathrm{~cm})$ box which was placed in a sound-proofed shielded cage. The animals were allowed 10-20 min to adapt themselves to the new environment prior to stimulation. The electroencephalogram (EEG) and afterdischarge (AD) were recorded on a polygraph. The hippocampus or amygdala was stimulated for $5 \mathrm{sec}$ with a square wave pulse (60 Hz in frequency. $1.0 \mathrm{msec}$ in duration). A stimulus of $80 \mu \mathrm{A}$ was given at first, and if $\mathrm{AD}$ was induced, a $60 "$ A stimulus was given. If $\mathrm{AD}$ was not induced by the $80 \mu \mathrm{A}$ stimulus. the stimulus intensity was increased by $20 \% \mathrm{~A}$ until the $A D$ was induced. After the threshold for AD was determined, electrical stimulation was performed with this intensity at intervals of $24 \mathrm{hr}$ until a kindling effect was established.

The development of clinical manifestations of kindling was measured and recorded under the following 4 stages: stage 1 : mouth movement and head nodding, stage 2: rearing, stage 3 : forelimb clonus with rearing. stage 4 : falling down.

Monoamine assay and histology: Rats were sacrificed by decapitation 14 days after the dorsal NA bundle or raphe nuclei lesion. The brains were quickly removed and placed on ice. The cerebral cortex, amygdala, striatum, hippocampus and hypothalamus were separated on an ice-cold glass plate according to the procedure described in our previous report (18). Each tissue was then weighed and assayed for NA, dopamine (DA), and $5-H T$. NA and DA were purified by the alumina adsorption method according to our previous report (19) and quantitated by the trihydroxyindole method of Chang (20). 5-HT was assayed by the method of Snyder et al. (21). The data obtained were evaluated using Student's $t$-statistics. The brainstem regions including the lesion site of the dorsal NA bundle or raphe nuclei were soaked in a $10 \%$ formalin solution for several days. They were sectioned coronally at a thickness of $80 \mathrm{\mu m}$ on a freezing microtome, and brain sections 
were stained with cresyl viloet to aid in the identification of the site and extent of the dorsal NA bundle or raphe nuclei lesion Figure 1 shows a representative section of a

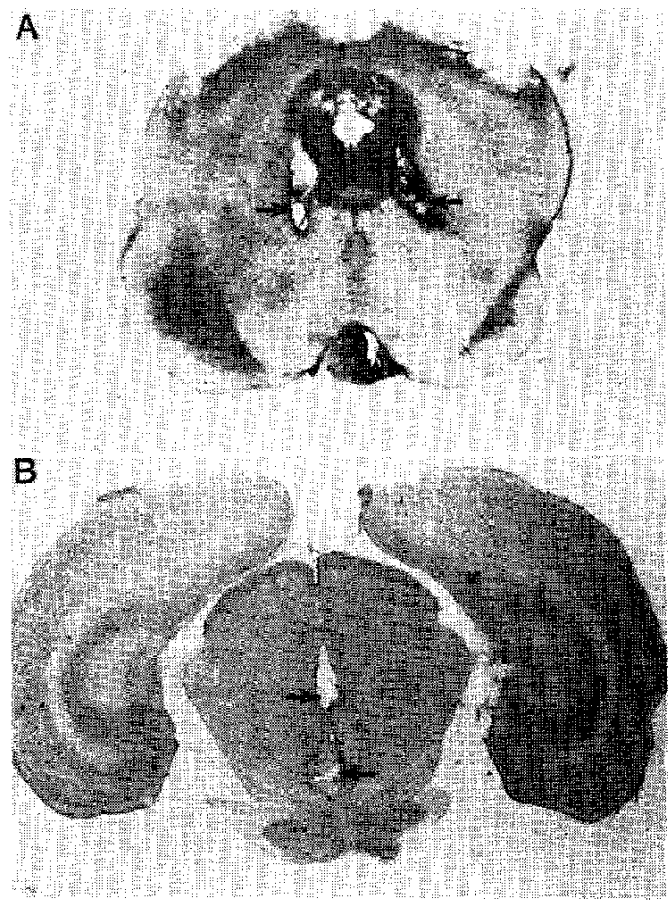

Fig. 1. Representative scotions of dorsat bunde lesions and raphe nuclei lesions. A: dorsal bundle lesions B: both dorsal and medial raphe lesions. typical dorsal NA bundle or raphe nuclei lesion.

\section{Results}

1. Dorsal NA bundle lesion: In hippocampal and amygdaloid kindling, successive daily stimulations elicited behavioral changes such as mouth movement, head nodding, rearing, forelimb clonus, and falling down in the sham operated and the dorsal NA bundle lesioned rats (Fig. 2).

In the hippocampal kindling. the mean numbers of stimulations required to reach stage 1,2, 3 and 4 in sham operated and dorsal NA bundle lesioned rats are shown in Table 1. The number of trials required for the establishment of kindling was significantly shortened by dorsal NA bundle lesion to $11.0 \pm 4.1$ trials for the dorsal NA bundle lesioned rats and $22.5 \pm 4.4$ trials for the sham operated rats $(\mathrm{P}<0.001)$. The number of trials required to reach stage 1 was most remarkably reduced by dorsal NA bundle lesions: $6.8 \pm 3.6$ trials were required in the dorsal NA bundle lesioned rats as compared with $16.7 \pm 3.9$ trials in the sham operated rats $(P<0.001)$. The high amplitude spike waves in the amygdala appeared earlier in the

Table 1. Changes in the hippocampal and amygdaloid kindling effects following the losion of dorsal noradrenaline bundle or midbrain raphe nuclei lesion

\begin{tabular}{|c|c|c|c|c|c|}
\hline \multirow[b]{3}{*}{ Treatment } & \multirow[b]{3}{*}{$N$} & \multicolumn{4}{|c|}{ Number of stimulation } \\
\hline & & Stage 1 & Stage 2 & Stage 3 & Stage 4 \\
\hline & & MeantS.D. & Mean土S.D. & Mean士S.D. & Meants.D. \\
\hline \multicolumn{6}{|l|}{ Hippocampal kindling } \\
\hline Sham lesion & 12 & $16.7 \pm 3.9$ & $20.3 \pm 4.8$ & $20.7 \pm 4.6$ & $22.5 \pm 4.4$ \\
\hline Dorsal bundle lesion & 8 & $6.8 \pm 4.1^{* * *}$ & $9.3 \pm 4.8^{* * *}$ & $9.8=4.7^{* *}$ & $11.0 \pm 4.2^{* * *}$ \\
\hline Sham lesion & 5 & $27.2=4.1$ & $29.4 \pm 5.4$ & $30.6 \pm 4.7$ & $32.4 \pm 4.0$ \\
\hline Raphe losion & 6 & $25.8 \pm 4.7$ & $28.8 \pm 6.0$ & $31.0=6.7$ & $33.7 \pm 7.1$ \\
\hline \multicolumn{6}{|l|}{ Amygdaloid kindling } \\
\hline Sham lesion & 6 & $4.3 \pm 1.7$ & $10.5 \pm 2.8$ & $13.7 \pm 2.9$ & $15.5 \pm 3.7$ \\
\hline Dorsal bundle lesion & 7 & $2.4 \pm 0.8^{*}$ & $4.6 \pm 1.8^{* * *}$ & $7.1=2.4^{* * *}$ & $9.0 \pm 1.5^{* *}$ \\
\hline Raphe nuclei lesion & 8 & $3.9 \pm 1.6$ & $9.3 \pm 3.4$ & $11.1 \pm 3.8$ & $15.6 \pm 4.6$ \\
\hline
\end{tabular}

${ }^{*} P<0.05,{ }^{* * P}<0.01,{ }^{* *} \mathrm{P}<0.001$ (2 tailed Student's $t$-test) 


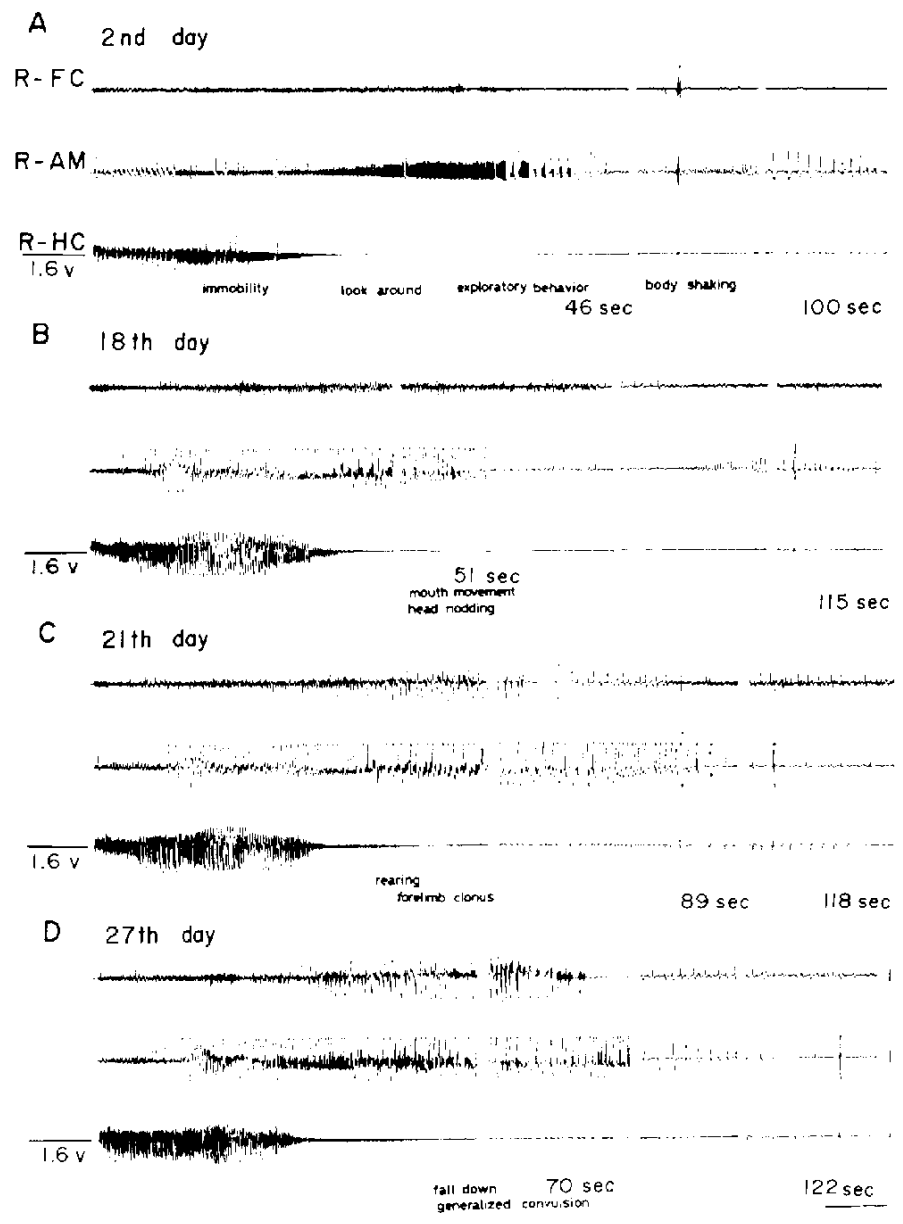

Fig. 2. Progressive changes of afterdischarge (AD) and behavioral convulsion elicited by nippocampal stimulation in a rat. A: the day of the second hippocampal stimulation, $B$ : the 18 th stimulation, $C$ : the 21 st stimulation, D: the 27 th stimulation, R-FC: right frontal cortex, R-AM: right amygdala, R-HC: right hippocampus. The time indicates when amygdaloid primary or secondary $A D$ ends. The vertical scale indicates $200 \mu \mathrm{V}$ and the horizontal scale $5 \mathrm{sec}$.

dorsal NA bundle lesioned animals than in the sham operated animals. No significant differences were shown in the number of trials required to reach stage 2, 3 and 4 between the dorsal NA bundle lesioned and the sham operated rats (Fig. 3).

In the amygdaloid kindling, the number of trials required to reach stage 4 was significantly shortened by dorsal NA bundle lesions. The average number of trials required to reach stage 4 was $9.0 \pm 1.5$ trials for the dorsal NA bundle lesioned rats and $15.5 \pm 3.7$ trials for the sham operated rats $(P<0.01$. Table 1). The number of trials required to reach stage 1 and stage 2 were most remarkably reduced by dorsal NA bundle lesions.

Contents of NA, DA and 5-HT in the cerebral cortex, amygdala, striatum, and hypothalamus are presented in Table 2. Cortical NA content was significantly lower in the dorsal NA bundle lesioned rats than that in the sham operated rats $(P<0.001)$. No significant differences were found in the other 


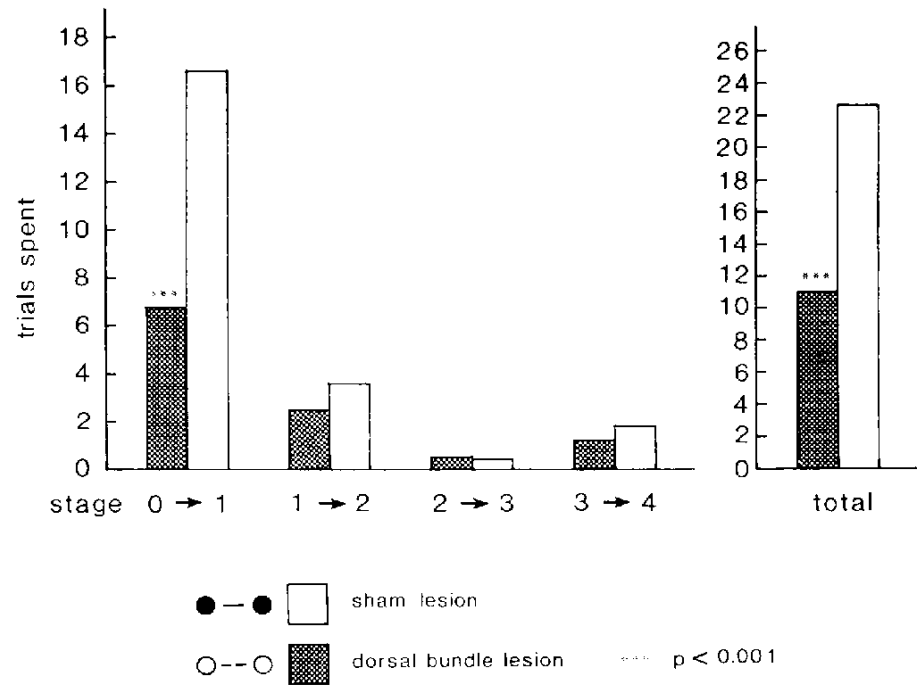

Fig. 3. The number of trials spent on each stage of kinding in the dorsal bundle lesioned rat. $0 \rightarrow 1$ : from stage 0 to stage $1,1 \rightarrow 2:$ from stage 1 to stage $2,2 \rightarrow 3:$ from stage 2 to stage $3,3 \rightarrow 4:$ from stage 3 to stage 4 . For the stage 0,1,2,3 and 4 see details in Methods. ** Significant different from sham lesion. (P<0.001, 2 tailed Student's t-test)

Table 2. Effects of dorsal noradrenaline bundle lesion on brain monoamine levels in rats

\begin{tabular}{lcc}
\hline Treatment & Sham lesion & Dorsal bundle lesion \\
Noradrenaline & $0.37 \pm 0.02$ & $0.10 \pm 0.02^{* * *}$ \\
Cortex & $0.42 \pm 0.08$ & $0.22 \pm 0.05$ \\
Amygdala & $2.00 \pm 0.23$ & $1.72 \pm 0.13$ \\
Hypothalamus & & \\
Dopamine & $7.71 \pm 0.75$ & $6.06 \pm 0.54$ \\
Striatum & $0.40 \pm 0.08$ & $0.67 \pm 0.12$ \\
Amygdala & & \\
Sorotonin & $0.31 \pm 0.04$ & $0.30 \pm 0.07$ \\
Cortex & $0.59 \pm 0.07$ & $0.49 \pm 0.14$ \\
Amygdala & $1.34 \pm 0.29$ & $1.38 \pm 0.18$ \\
Striatum & $1.16 \pm 0.25$ & $0.71 \pm 0.20$ \\
Hypothalamus & &
\end{tabular}

Rats were sacrified 14 days after the dorsal noradrenaiine bundle lesion. Each value is the mean $\pm S$.E. ( $\mu \mathrm{g} / \mathrm{g}$ tissue). ** Significantly different from sham lesion ( $P<0.001,2$ tailed Student's $t$-test)

regions between the sham operated and the dorsal NA bundle lesioned rats in NA, DA and $5-H T$ contents.

2. Raphe nuclei lesion: The behavioral and electroencephalographic patterns of the hippocampal and amygdaloid kindling in the sham operated and the raphe nuclei lesioned rats agreed with results in non-lesioned rats in our previous experiment (22). No significant difference was found between the raphe nuclei lesioned and the sham operated rats in the number of stimulations required to 
Table 3. Effects of midbrain raphe nuclei lesion on brain monoamine levels in rats

\begin{tabular}{lcc}
\hline Treatment & Sham lesion & Raphe nuclei lesion \\
Site & & - \\
\hline Noradrenaline & $0.41 \pm 0.06$ & $0.33 \pm 0.05$ \\
Cortex & $0.53 \pm 0.03$ & $0.52 \pm 0.10$ \\
Amygdala & $0.48 \pm 0.03$ & $0.30 \pm 0.01^{* *}$ \\
Hippocampus & & \\
Serotonin & $0.40 \pm 0.03$ & $0.14 \pm 0.01^{* *}$ \\
Cortex & $0.78 \pm 0.06$ & $0.19 \pm 0.05^{* * *}$ \\
Amygdala & $0.53 \pm 0.06$ & $0.22 \pm 0.06^{* *}$ \\
Hippocampus &
\end{tabular}

Rats were sacrified 14 days after the midbrain raphe nuclei lesion. Each value is the mean $1 S . E$. ( $\mu \mathrm{g} / \mathrm{g}$ tissue). $\quad{ }^{* *} P<0.01,{ }^{* *} P<0.001$ (2 iailed Student's $t$-tost)

reach stages 1, 2, 3 and in the total number of trials required for the establishment of the hippocampal and amygdaloid kindling effects (Table 1).

The contents of 5-HT and NA in the cerebral cortex, amygdala, and hippocampus are presented in Table 3. Cortical and amygdaloid 5-HT contents were significantly lower in the raphe nuclei lesioned rats than in the sham operated rats $(P<0.001)$. A significant reduction was also observed in the content of hippocampal 5-HT and NA in the raphe nuclei lesioned rats $(P<0.07)$. No significant difference was found in the other regions between the raphe nuclei lesioned rats and the sham operated rats in the contents of NA and $5-H T$.

\section{Discussion}

The results obtained in the present experiment showed that dorsal NA bundle lesions markedly facilitated the formation of the hippocampal kindling effect. Especially, the most significant reduction was seen in the number of trials required to reach stage 1 . These facts suggest that the central noradrenergic mechanism plays an inhibitory role in the development of hippocampal kindling. Clinical signs of stage 1 are mouth movement and head nodding. It is known that oral movement is easily evoked by electrical stimulation of the amygdaloid nucleus (23). The development of behavioral manifestations in the hippocampal kindling was similar to that in the amygdaloid kindling. although more stimulations were required to establish the kindling effect in the former. Furthermore, the development of behavioral changes in the hippocampal kindling coincided with the increasing amplitude of spike waves in the amygdaloid lead (22). The functional anatomical relationships between the amygdala and hippocampus have been well demonstrated (24). The ventral hippocampal stimulation evokes field potentials with a short latency throughout the amygdaloid nucleus (25). A strong propagation of afterdischarge activity occurs between the two structures (26). Therefore, it is quite conceivable that the amygdala plays an important role in the formation of the hippocampal kindling effect.

Dorsal NA bundle lesions also facilitated the establishment of amygdaloid kindling. This is in good agreement with the finding by Ehlers et al. (8) that the transection of the forebrain noradrenergic pathway facilitates the formation of the amygdaloid kindling effect. In the present experiment, cortical NA content was assayed as an index for estimating the extent of dorsal NA bundle lesion since the diffuse innervation of NA 
nerves through the dorsal NA bundle exists in the neocortex (27). The animals with dorsal NA bundle lesions showed an approximately $70 \%$ decrease in the cortical NA content, indicating that the greater part of the dorsal NA bundle was destroyed in this experiment.

Corcoran et al. (6) reported that rats with a selective depletion of forebrain NA displayed a highly significant facilitation of amygdaloid kindling. They found that noradrenaline depletion failed to affect either the primary site threshold for afterdischarges or the duration of the initial afterdischarges evoked in the primary site. Therefore, they postulated that the facilitatory effects of NA depletion in the amygdaloid kinding were apparently related to disinhibition of the spread of seizure discharges from the stimulated site to the other brain areas rather than to increased epileptogenesity in the stimulated site itself. In our present experiment, there were no significant differences in the duration of afterdischarges elicited by the first hippocampal stimulation between the dorsal NA bundle lesioned and the sham operated animals. In hippocampal kindling, however, the high amplitude spike waves in the amygdala appeared earlier in the dorsal NA bundle lesioned animals than in the sham operated animals. As mentioned before, the most significant decrease is seen in the number of trials spent to reach stage 1 , and the sign of stage 1 is mouth movement which is easily evoked by the electrical stimulation of the amygdala (23). Therefore, it is conceivable that the facilitatory effect of NA depletion on the development of the hippocampal kindling effect is related to disinhibition of the spread of seizure discharges from the hippocampus to the amygdala. Furthermore, in rats with NA depletion by dorsal NA bundle lesions, the amygdala may become readily excitated by electrical stimulation of the hippocampus; and facilitation of the spread of seizure discharges from the amygdala to the other brain structures would also occur.

On the other hand, raphe nuclei lesions did not have any effect on the formation of the hippocampal kindling effect. Raphe lesioned rats showed an approximately $65 \%, 75 \%$ and $60 \%$ decrease in the cortical, hippocampal, and amygdaloid 5-HT contents, respectively.

The role of brain 5 -HT systems in the formation of the kindling effect has not yet been well clarified. Kovaks and Zoll (10) reported that electrical stimulation of the median raphe nucleus blocked kindled amygdaloid seizures induced by threshold stimulation. Racine and Coscina (11) reported that both dorsal and medial raphe lesions facilitated the development of amygdaloid kindling: however, systemic injection of $\mathrm{p}$ chlorophenylalanine (pCPA), a tryptophan hydroxylase inhibitor, retarded the development of convulsions by amygdaloid stimulation. In view of the conflicting data, Racine and Coscina (11) proposed that 5-HT systems contain a mixture of excitatory and inhibitory subsystems, which is supported by the findings from studies employing iontophoretic application of $5-\mathrm{HT}$ to single neurons (28). Therefore, it is considered that the influence of disrupting brain 5- $\mathrm{HT}$ neurons on various seizures may depend upon the balance of activity remaining in these subsystems. This explanation may account for the discrepancy between our present results and others. In the present experiment, none of the rats undergoing raphe nuclei lesions showed significant changes in the formation of the hippocampal kindling effect. Whatever the exact role of brain 5-HT neurons may be in the formation of the hippocampal kindling effect. this fact seems to indicate that they are not robust.

The present results suggest that the dorsal NA bundle system plays an inhibitory role in the development of the seizure discharges 
and the behavioral convulsions in hippocampal kindling.

\section{References}

1) Goddard, G.V.. Mclntyre, D.C. and Leech, C.K.: A permanent change in brain function resulting from daily electrical stimulation. Exp. Neurol. 25, 295-330 (1969)

2) Callaghan, D.A. and Schwark, W.S.: Involvement of catecholamines in kindled amygdaloid convulsions in the rat. Neuropharmacology 18 , $541-545$ (1979)

3) Taber, K.H., McNamara, J.J. and Zornetzer, S.F.: Status epilepticus: A new rodent model. Electroencephalogr. Clin. Neurophysiol. 43, 707-724 (1977)

4) McIntyre, D.C.: Amygdala kindling in rats: Facilitation after local amygdala norepinephrine depletion with 6-hydroxydopamine. Exp. Neurol. 69, 395-407 (1980)

5) Arnold, P.S., Racine, R. and Wise, P.A.: Effects of atropine, reserpine. 6-hydroxydopamine and handling on seizure development in the rat. Exp. Neurol. 40, 457-470 (1973)

6) Corcoran, M.E. and Mason, S.T.: Role of forebrain catecholamines in amygdaloid kindling. Brain Res. 190, 473-484 (1980)

7) Engel, J., Jr. and Kotzman, R.: Facilitation of amygdaloid kindling by lesions of the stria terminalis. Brain Res. 122, 137-142 (1977)

8) Ehlers, C.E., Clifton, D.K. and Sawyer, C.H.: Facilitation of amygdala kindling in the rat by transecting ascending noradrenergic pathways. Brain Res. 189, 274-278 (1980)

9) De la Torre. J.C., Kawanaga, H.M. and Mullan, S.: Seizure susceptibility after manipulation of brain serotonin. Arch. Int. Pharmacodyn. Ther. 188, 298-304 (1970)

10) Kovaks, D.A. and Zoll, J.G.: Seizure inhibition by median raphe nucleus stimulation in rat. Brain Res. 70, 165-169 (1974)

11) Racine, R. and Coscina, D.V.: Effects of midbrain raphe lesions on systemic p-chlorophenylalanine on the development of kindled seizures in rats. Brain Res. Bull. 4, 1-7 (1979)

12) Siegel, J. and Murphy, G.J.: Serotonergic inhibition of amygdala-kindled seizures in cats. Brain Res. 174, 337-340 (1979)

13) Racine, .R.: Kindling, the first decade. Neurosurgery 3, 234-252 (1978)

14) Curtis, D.R. and Crawford, G.M.: Central synaptic transmission : microelectrophoretic studies. Annu. Rev. Pharmacol. Toxicol. 9, 209-240 (1969)
15) Gastaut, H., Naquet, R., Meyer, A., Caraugh, J.B. and Becke, E.: Experimental psychomotor epilepsy in the cat : electroclinical arıd anatomicopathological correlations. J. Neuropathol. Exp. Neurol. 18, 270-293 (1959)

16) De Groot, J.: The forbrain in stereotaxic coordinates. Verch. K. Acad. Wet. 52, 1-40 (1959)

17) König, J.F.R. and Klippel, R.A.: The Rat Brain: A Stereotaxic Atlas. Williams and Wilkins. Baltimore (1963)

18) Oishi, R. and Ueki, S.: Facilitation of muricide by dorsal norepinephrine bundle lesions in olfactory bulbectomized rats. Pharmacol. Biochem. Behav. 8, 133-136 (1978)

19) Razzack, A., Fujiwara, M., Oishi, R. and Ueki, S.: Possible involvement of a central noradrenergic system in automutilation induced by clonidine in mice. Japan. J. Pharmacol. 27, 145-152 (1977)

20) Chang, C.C.: A sensitive method for spectrophotofluorometric assay of catecholamines. Int. J. Neuropharmacol. 3, 643-649 (1964)

21) Snyder, S.H., Axelrod, J. and Sweig, M.: A sensitive and specific fluorescence assay for tissue serotonin. Biochem. Pharmacol. 14, 831835 (1965)

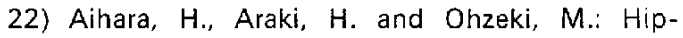
pocampal kindling and effect of antiepileptic drugs. Japan. J. Pharmacol. 32, 37-45 (1982)

23) Boldwin, M., Frost, L.L. and Wood, C.D.: Investigation of the primate amygdala. Movement of the face and jaw. Neurology (N.Y.) 4, 586-598 (1954)

24) Morrison, F. and Polett, C.E.: Hippocampal influence on amygdala unit activity in awake squirrel monkeys. Brain Res. 192, 353-369 (1980)

25) Heath, R.G.: Brain function in epilepsy: midbrain medullary, and cerebellar interaction with the rostral forebrain. J. Neurol. Neurosurg. Psychiatry 39, 1037-1051 (1976)

26) Kaada, B.R.: Stimulation and regional ablation of the amygdaloid complex with reference of functional representations. In The Neurobiology of the Amygdala, Edited by Eleftheriou. B.E., p. 205-282. Plenum, New York (1972)

27) Lindvall, $O$. and Bjorklung, A.: The organization of the ascending catecholamine neuron systems in the rat brain. Acta Physiol. Scand. Supp. 412, 1-48 (1974)

28) Bradley, P. and Dray, A.: Modification of the response of brainstem neurones to transmitter substances by anaesthetic agents. Br. J. Pharmacol. 48, 212-224 (1973) 